\title{
A NEW MODEL FOR ACHIEVING VALUE ADDED GOALS IN A COLLABORATIVE INDUSTRIAL SCENARIO
}

\author{
Giuseppe Confessore ${ }^{1}$, Giacomo Liotta ${ }^{1,2}$, Silvia Rismondo ${ }^{1}$ \\ 'Istituto di Tecnologie Industriali e Automazione \\ Consiglio Nazionale delle Ricerche \\ Area della Ricerca Roma Tor Vergata, Via Fosso del Cavaliere, 100 \\ 00133 Roma - ITALY \\ g.confessore@itia.cnr.it,g.liotta@itia.cnr.it,s.rismondo@itia.cnr.it \\ ${ }^{2}$ Dipartimento di Ingegneria dell'Impresa \\ Università di Roma "Tor Vergata" \\ Via del Politecnico 1-00133 Roma-ITALY.
}

\begin{abstract}
Nowadays, at industrial research level, seems to be recognised that the collaboration among firms is becoming a relevant and effective way of operating. For the enterprises rather than compete on costs it could be necessary increase the product/service value added; this goal could be effectively achieved by collaborating with other firms in the value chain. In this preliminary work, we provide a model formulation for supporting the potential decision of getting new business opportunities through the exploitation of a competence-based collaborative advantage.

We propose a model formulation for maximising the potential value added generated by the convergence of complementary competences in the inter-firm collaboration. For this purpose we assume as given a codified set of competences and for each one of them a codified benchmark value.
\end{abstract}

\section{INTRODUCTION}

Nowadays, at industrial research level, seems to be recognised that the collaboration among firms is becoming a relevant and effective way of operating (e.g., see for instance Camarinha-Matos (1999) and Kaihara and Fujii (2002)). In particular, the strong need of new forms of collaboration is necessary consequence of the increasing competition in the industrial scenario mainly due to the emergent markets, such as the Asiatic one, and the new plants' locations, that force the enterprises worldwide to adopt, for instance, new procurement, production or distribution strategies. In this scenario, for the enterprises rather than compete on costs it could be necessary increase the product/service value added; this goal could be effectively achieved by collaborating with other firms in the value chain. In fact, the latest research literature concerning the inter-organizational network field, demonstrates the relevance of knowledge-based (Conner and Prahalad, 1996) and 
dynamic capability (Teece, Pisano and Shuen, 1997) theories, both based on the enterprises competence concept.

Despite the increasing interest on this research field, still a lack can be noticed in terms of models describing the collaboration and thus considering the relevance of competence as a way to achieve the value added goal. On the other hand, the definition of a model could be useful for understanding which could be, in practice, the potential business opportunities generated by the collaboration.

In fact, this preliminary work aims at defining a model formulation for understanding whether a network of collaborative enterprises has the competences for realizing and sustaining a new emerging business process.

The paper is organized as follows. Section 2 refers to related works supporting the adopted approach. Section 3 describes a business process in terms of the activities to be done; secondly, it considers a set of competences and describes the map linking the competences, the activities, and the actors of the collaboration. Finally, Section 4 provides a mathematical formulation and a short example.

\section{RELATED WORKS}

The presented model introduces the competence concept, that Chung, Yam, and Chan (2004) define as one of the four main drivers for collaboration (e.g., the collaborative advantage, the regional advanced, the innovation capacity -knowledge resource- and the competences). Moreover, it assumes a business process consisting of a set of (interdependent) activities, and through the paper it has been considered as a competence the company's capability in executing, for instance, the process planning, the product/service design and implementation, the distribution, or the company's productive and elaborative capacity, then it considers both quantitative and qualitative aspects.

In this setting, the presented paper proposes an approach for assigning the activities that cover an entire inter-organisational business process to the collaborative enterprises and, concurrently, for maximising the potential value added generated by the convergence of complementary competences in the inter-firm collaboration.

For this purpose, through the paper is assumed as given a codified set of competences and for each one of them a codified benchmark value. Thus, the actors could establish in a consistent way their ability w.r.t. a codified competence. Given these (assumed) codes, a map which links the activities and the competences required to perform them is exploited. In fact, it can be assumed that, for achieving the product/service value added goals, it is necessary to concentrate on the company's capabilities while providing the most promising combination of available competences. A similar approach for codifying the level of expertise into a generic competence frame is presented in Hammami, Burlat, and Champagne (2003). When the authors define a technological map underlying a product, they introduce $(i)$ a product attainment graph (i.e. logical sequence of production and administrative activities), and (ii) an activities/competencies map. They then present a competencies map for each enterprise of the network for the standardization of the skills' description. Therefore, this paper also defines a map linking the activities and the competences required. 
Concerning the data and knowledge sharing within the network, privacy and autonomy needs of participants can impact on the full effectiveness of the collaboration. These relevant factors are in practice possible barriers to the interorganisational cooperation when the degree of process complexity and the number of transactions increase. Models and technology solutions have been designed for meeting cooperative process management, coordination, integration, privacy needs in inter-organisational business processes, as explained in Perrin and Godart (2004) where the authors provide a model and an architecture for supporting the realization of a collaborative scenario for multi-enterprise business processes.

Interesting case studies concerning corporate collaborative networks are presented in Danilovic and Winroth (2005). Noticeable findings of the reported projects' experiences, among others, concern the increasing level of competences of participating partners, important prerequisites (e.g. openness, trust, and enabling IT resources), investments in time and resource, form of collaboration (influenced by the collaboration objectives), legal aspects. The authors point out the relevance of identifying and analysing the pre-requisites for inter-organisational integration in network settings; they also provide a tentative framework for this purpose.

Also in Carbonara, Giannoccaro, and Potrandolfo (2002), the authors underline the relevant role of the complementary competences (technical and organisational) for very interesting forms of collaboration that take place in the Industrial Districts (i.e. SMEs located and integrated in a geographical area and specialised in complementary production processes or services) when a strategy of external growth is addressed.

\section{PROBLEM AND STATEMENT DEFINITION}

Given a set of enterprises joining the network, the goal is to allocate among the actors the necessary activities for fulfilling a new emerging business process while maximising the product/service value added. We assume that the value added increases by considering the concept of actors competence (or capability) in carrying out the activities of a business process.

In this setting, $(i)$ an industrial scenario involving a set $E$ of $z$ enterprises, $E=$ $\left\{e_{1}, \ldots, e_{z}\right\}$ and ( $\left.i i\right)$ a business process $P$ consisting of a set $A$ of $k$ activities $A=\left\{a_{1}\right.$, $\left.\ldots, a_{k}\right\}$ have been considered; technological constraints are given forcing each activity to start its execution after the completion time of its predecessors. In the operations research literature, considering a centralized decision-maker, the problem of computing the starting time of the activities respecting the precedence constraints, and minimizing the completion time of all them, is a well known scheduling problem [6]. The computational complexity of this problem increases when a set $R$ of $h$ finite capacity resources is given (i.e., $R=\left\{r_{1}, \ldots, r_{h}\right\}$ ), and for each activity $a_{i}$ a specific resource requirement $v_{i w}$ is defined (i.e., for all $a_{i}, w \in R$, and $0 \leq v_{i w} \leq r_{w}$ ). Under this assumption the scheduling problem is characterized by both technological and resource constraints. Classical models and resolution approaches are based on the strong assumption that a centralized decision-making process is given and all the information about resource requirements and technological constraints are known. 
Since this work considers an inter-organizational framework, that is a set of (independent) enterprises involved in the execution of a specific collaborative business process is given (that, clearly, could not be modelled as a centralized decision maker), more probably it is known which competence is required to perform the activities rather than the exact set of resources for executing them.

This is one of the main issue of this paper; in fact, in the collaborative perspective, since the information sharing is a crucial issue, in this work the enterprises have not to share their internal data or specialized knowledge (e.g., trust and privacy issues could arise). Companies have to declare what are able to perform and which is their corresponding capability. Actors' capabilities are codified and classified through common benchmark values (assumed as given and accepted within the network).

Nevertheless, the presented preliminary model, that is formalized in the following Section, could be useful for determining commitments and responsibilities among members of a collaborative network when a new business opportunity arise. For this purpose it relies on a competence-based analytical approach for identifying the complementary competences that allow to consider an high value added objective to be pursued through the collaboration.

\section{THE MODEL DEFINITION}

This work addresses the problem of assigning the activities of a business process to collaborative enterprises while maximising the competence value, thus, in the current perspective, maximising the product/service value added. It assumes that the actors always have the availability to carry out the assigned activities, and it assigns each activity to at most one of the enterprises (e.g., the activities cannot be done simultaneously by two or more actors).

The following sets and parameters can be introduced:

- The set $C$ of $w$ competences $C=\left\{c_{1}, \ldots, c_{w}\right\}$ globally accepted between the collaborative enterprises.

- The $k$ sub-sets $C_{1}, \ldots, C_{k}$ representing the set of competences required for the execution of each activity in $A$ (i.e., at each $a_{i} \in A$ is associated a sub-set $C_{i} \subseteq$ C).

- The $z$ sub-sets $\widetilde{C}_{j} \subseteq C$ representing the set of competences declared by each enterprise $e_{j}$ according to the standard codes.

- The parameters $\varepsilon_{j h}$ providing the degree of capability of enterprise $e_{j}$ respect to a specific competence $c_{h}$. $\varepsilon_{j h}$ is a positive value in $[0,1]$, where 0 means $e_{j}$ has not the competence $h$, and $\varepsilon_{j h}=1$ means $e_{j}$ has the competence $h$; the value $\varepsilon_{j h}$ is defined by using a common scale of values (accepted by the collaborative actors).

\subsection{Model hypothesis and assumptions}

The following hypothesis can be formulated:

- Each sub-set $C_{i}$ can be modelled as a binary vector $u\left(a_{i}\right)$ composed of $w$ elements, where the $h$-th element (i.e., $\left.u_{h}\left(a_{i}\right)\right)$ is equal to 0 if activity $a_{i}$ does not require the competence $c_{h}$ for being executed, and is equal to 1 otherwise. 
- Each sub-set $\widetilde{C}_{j}$ can be modelled as a vector $v\left(e_{j}\right)$ composed of $w$ elements, where the $h$-th element (i.e., $v_{h}\left(e_{j}\right)$ ) is equal to 0 if the enterprise $e_{j}$ has not the competence $c_{h}$, and is equal to a positive value $\varepsilon_{j h}$ otherwise. In particular, $\varepsilon_{j h}$ represents the capability of $e_{j}$ w.r.t. a specific competence framework as mentioned in Section 2.

It can be emphasized that the two vectors are consistent since $u\left(a_{i}\right)$ represents exactly the competences required for executing $a_{i}$ while assuming the capability respect each competence $c_{h} \in C$ equal to 1 or 0 , that is considering the same scale of values of $\varepsilon_{j h}$ (where $c_{h}$ equal to 1 is exactly the maximum (desired) value).

Furthermore, the definition of the two vectors $u\left(a_{i}\right)$, and $v\left(e_{j}\right)$ is required since this work mainly focuses on two relevant aspects: (1) which enterprise has the competences for carrying out one activity; (2) which level of performance each enterprise could deploy in executing a specific activity. Therefore, it is indispensable to measure how much an enterprise fits the competences required for executing, and then performing as best, a specific activity, thus it is required to measure which is the 'distance' between the vectors. In order to calculate this distance value, it is necessary to introduce the vector $g\left(a_{i}, e_{j}\right)$ of $w$ elements where the $h$-th element is computed as follows: $g_{h}\left(a_{i}, e_{j}\right)=\min \left\{u_{h}\left(a_{i}\right), v_{h}\left(e_{j}\right)\right\}$. Vector $g\left(a_{i}, e_{j}\right)$ extracts from vector $v\left(e_{j}\right)$ exactly those elements that match the corresponding elements of $u\left(a_{i}\right)$ being equal to 1 . In other words, it catches the competences of $e_{j}$ respect to those ones required by a given activity $a_{i}$.

Given the activity $a_{i}$, it is possible to compute for each $e_{j} \in E$ the vectors $g\left(a_{i}, e_{j}\right)$, and calculate the Euclidean distance between $g\left(a_{i}, e_{j}\right)$, and $u\left(a_{i}\right)$. Below is the standard Euclidean distance formula between vectors $g\left(a_{i}, e_{j}\right)$ and $u\left(a_{i}\right)$.

$$
\operatorname{euc}\left(g\left(a_{i}, e_{j}\right), u\left(a_{i}\right)\right)=\left(\sum_{h=1}^{k}\left(g_{h}\left(a_{i}, e_{j}\right)-u_{h}\left(a_{i}\right)\right)^{2}\right)^{\frac{1}{2}}
$$

Due to the distance function, the following parameters are introduced:

$$
\omega_{i j}=e u c\left(g\left(a_{i}, e_{j}\right), u\left(a_{i}\right)\right)
$$

Each $\omega_{i j}$ represents the capability of $e_{j}$ w.r.t. the execution of the activity $a_{i}$.

\subsection{The optimization model}

The model considers the binary variable $x_{i j}$ equal to 1 , if it assigns the activity $a_{i}$ to the enterprise $e_{j}$ and 0 otherwise, for all $e_{j} \in E, a_{i} \in A$, and the problem can be formalized as follows:

$$
\begin{array}{ll}
\min \sum_{i \in A} \sum_{j \in E} \omega_{i j} x_{i j} & \\
\text { s.t. } & \forall i \in A \\
\sum_{j \in E} x_{i j}=1 & \forall j \in E, \forall i \in A \\
x_{i j} \in\{0,1\} &
\end{array}
$$

Then the model aims at assigning each activity to exactly one enterprise (constraints 1), while minimising the distance among vectors that is obtaining the solution maximising the value added (e.g., competences value). 
This is an assignment problem that can be referable to a matching problem; in fact, some enterprises can perform more than one activity. Such multiple assignments can be modeled by simply replicating the enterprises vertices, of the classical bipartite graph, as many times as the number of activities they can handle.

Clearly, this model considers a centralized-decision maker, that is able to collect the enterprises' degree of competence w.r.t. each activity, and to find the best solution. In this setting, it can be remarked that the enterprises have not to share their proper data (that is one of the main motivation of this preliminary work), but they only provide, and then share, the aggregate values $\varepsilon_{j h}$.

\subsection{A model example}

In the next, a short example is given in order to describe some potentialities of the presented model. In fact, although big enterprises could easily demonstrate which is their core business and their competences in performing specific activities, this could not be so easy, in practice, for the SMEs. A SME could not have the same responsiveness to a specific market request/requirement, but two collaborating SMEs could have it. Due to this fact, this paper provides answers to several questions such as, for instance, how the SMEs can work together for achieving common goals, and how they could be competitive in doing that. For this purpose, a very simple example is given, even if future works will concern the application of the given model to real case-studies.

Suppose there is a set of $z=2$ SMEs operating in the high-tech sector, and aiming at collaborating for commercial goals; the first one manufactures silicon base for hardware-oriented customers while cutting and preparing the silicon base for molding. It is focused on front-end processes as wafer fabrication and probe. The second one is specialized in data circuit molding thus it is focused on back-end processes as assembly and final test. Clearly, they are on the value chain but they have never collaborated since each one of them has its own specific customers.

Suppose they observe a new market request consisting in producing a new data circuit based on a new silicon base shape. Then, the new business process consists in procuring the row materials for the silicon base production and then in providing the data circuit molding. In particular, four main macro-activities can be defined for fulfilling the emerging business opportunity: (1) procurement; (2) production; (3) assembly and test; (4) delivery.

The specific SMEs core businesses do not allow to meet directly the market needs. They only could address the market being suppliers of big enterprises that work as a market leader. On the other hand, if they collaborate they could exploit both complementary and concurrent competences.

For the sake of simplicity, suppose the activities execution requires eight main competences: (1) bargaining power w.r.t. suppliers; (2) negotiation capability; (3) production planning; (4) production capacity; (5) assembly; (6) test tools; (7) delivery planning; (8) transportation capacity. In this example, the SMEs' plants are supposed to be close to each other (i.e., so doing no transport activities have to be considered).

Therefore, the business process $P$ is composed of four activities $a_{1}, a_{2}, a_{3}, a_{4}$ (i.e., $k$ $=4)$, and is given a global accepted code of $w$ competences, $C=\left(c_{1}, c_{2}, c_{3}, c_{4}, c_{5}\right.$, 
$c_{6}, c_{7}, c_{8}$ ) (i.e., $w=8$ ). Each enterprise expresses its degree of competence w.r.t. the scale of values, thus previous information, in synthesis, can be modelled as follows:

1. $u\left(a_{1}\right)=[1,1,0,0,0,0,0,0], u\left(a_{2}\right)=[0,0,1,1,0,0,0,0], u\left(a_{3}\right)=[0,0,0$, $0,1,1,0,0]$, and $u\left(a_{4}\right)=[0,0,0,0,0,0,1,1]$ represent the vectors of competences required for $a_{1}, a_{2}, a_{3}$, and $a_{4}$, respectively.

2. $v\left(e_{1}\right)=[0.3,0.8,0.1,0.8,0.3,0.4,0.6,0.6]$, and $v\left(e_{2}\right)=[0.6,0.1,0.5,0.7$, $0.8,0.9,0.4,0.9]$ represent the competence vectors of $e_{1}$, and $e_{2}$, respectively.

Given the vectors mapping the competences, for each $e_{j}$, and for each $a_{i}$, the vectors $g\left(a_{i}, e_{j}\right)$ can be computed.

$$
\begin{array}{ll}
g\left(a_{1}, e_{1}\right)=[0.3,0.8,0,0,0,0,0,0] & g\left(a_{3}, e_{1}\right)=[0,0,0,0,0.3,0.4,0,0] \\
g\left(a_{1}, e_{2}\right)=[0.6,0.1,0,0,0,0,0,0] & g\left(a_{3}, e_{2}\right)=[0,0,0,0,0.8,0.9,0,0] \\
g\left(a_{2}, e_{1}\right)=[0,0,0.1,0.8,0,0,0,0] & g\left(a_{4}, e_{1}\right)=[0,0,0,0,0,0,0.6,0.6] \\
g\left(a_{2}, e_{2}\right)=[0,0,0.5,0.7,0,0,0,0] & g\left(a_{4}, e_{2}\right)=[0,0,0,0,0,0,0.4,0.9]
\end{array}
$$

Then, the Euclidean distance is calculated, as follows:

$$
\begin{array}{ll}
\text { euc }\left(g\left(a_{1}, e_{1}\right), u\left(a_{1}\right)\right)=\omega_{11}=0.73 & \text { euc }\left(g\left(a_{3}, e_{1}\right), u\left(a_{3}\right)\right)=\omega_{31}=0.92 \\
\text { euc }\left(g\left(a_{1}, e_{2}\right), u\left(a_{1}\right)\right)=\omega_{12}=0.98 & \text { euc }\left(g\left(a_{3}, e_{2}\right), u\left(a_{3}\right)\right)=\omega_{32}=0.22 \\
\text { euc }\left(g\left(a_{2}, e_{1}\right), u\left(a_{2}\right)\right)=\omega_{21}=0.92 & \text { euc }\left(g\left(a_{4}, e_{1}\right), u\left(a_{4}\right)\right)=\omega_{41}=0.57 \\
\text { euc }\left(g\left(a_{2}, e_{2}\right), u\left(a_{2}\right)\right)=\omega_{22}=0.58 & \text { euc }\left(g\left(a_{4}, e_{2}\right), u\left(a_{4}\right)\right)=\omega_{42}=0.61
\end{array}
$$

Therefore, the model is as follows:

$\min 0.73 x_{11}+0.98 x_{12}+0.92 x_{21}+0.58 x_{22}+0.92 x_{31}+0.22 x_{32}+0.57 x_{41}+0.61 x_{42}$ s.t.

$$
\begin{array}{ll}
x_{11}+x_{12}=1 & x_{31}+x_{32}=1 \\
x_{21}+x_{22}=1 & x_{41}+x_{42}=1 \\
x_{i j} \in\{0,1\} \forall i \in A, \forall j \in E &
\end{array}
$$

The solution is: $x_{11}=1, x_{12}=0, x_{21}=0, x_{22}=1, x_{31}=0, x_{32}=1, x_{41}=1, x_{42}=0$ and corresponds in the assignment of the activity $a_{1}$ to $e_{1}, a_{2}$ to $e_{2}, a_{3}$ to $e_{2}$, and $a_{4}$ to $e_{1}$. The objective function value is 2.1 .

Although this example can not be exhaustive, it shows how, by preliminary competence evaluation (the distance measuring the enterprises capability w.r.t. the activities), the model could suggest the activities allocation for assuring the best outcome in terms of competences maximization. Clearly, the model will be useful for the collaborative enterprises that aim at fulfilling emerging business processes where, probably, a predefined allocation does not exist. Furthermore, the model could be especially useful when increase either the activities detail, the competence detail, and, obviously, the number of enterprises potentially involved in the business process. 


\section{CONCLUSIONS}

This paper assumes that a way for achieving value added goals in delivering products and services worldwide, the enterprises have to concentrate on their competences.

It proposes a competence-based analytical model for allocating tasks among the actors of a collaborative network in order to potentially fulfil the processes related to high value added products or services. The complementary competences exploitable by the network are maximised while taking into account assignment constraints. So doing the model establishes a direct connection between the competences required for executing the tasks referred to an entire business process and the value added that can be generated by the proper allocation of activities.

Through the preliminary model only possible commitments and responsibilities throughout a complete business process can be determined without generating privacy and autonomy problems among the network's participants. Hence, the proposed approach could be useful for supporting the decision of getting new business opportunities through the collaboration.

The model relevance could increase when it is necessary to consider additional model constraints such as the enterprise temporal availability in carrying out the assigned activity. Due to this fact, future works will enhance the model while taking into account for instance costs, time, and product/service quality. Further, the model will be tested on real industrial settings.

\section{REFERENCES}

1. Camarinha-Matos, L.M., and H. Afsarmanesh. The virtual enterprise concept, in Infrastructures for Virtual Enterprises. In: Luis, Afsarmanesh H (eds.): Kluwer Academic Publisher; 3-14, 1999.

2. Carbonara, N., Giannoccaro, I., Potrandolfo, P. Supply Chain within industrial districts: A theoretical framework.. In Intemational Joumal of Production Economics; 76: 159-176, 2002.

3. Chung, W.C., Yam, A.Y.K., Chan M.F.S.. Networked enterprise: a new business model for global sourcing. In Intemational Journal of Production Economics; $87: 267-280,2004$.

4. Conner, K.R, and C.K. Prahalad, A Resource-Based Theory of the Firm: Knowledge vs. Opportunism, In Organization Science 7, 1996.

5. Danilovic, M., Winroth, M. A tentative framework for analysing integration in a manufacturing network settings: a case study. In Journal of Engineering and Technology Management; $22: 141$ $158,2005$.

6. Demeulemeester, E.L., W.S. Herroelen. Project Scheduling: A Research Handbook. Kluwer 2002.

7. Hammami, A., P. Burlat, and J.P. Champagne. Evaluating orders allocation within networks of firms. In International Journal of Production Economics; $86: 233-249,2003$.

8. Kaihara, T., Fujii S... A proposal on negotiation methodology in VE, Collaborative Business Ecosystems and Virtual Enterprises, Kluwer Academic Publishers, Boston; pp. 125-132, 2002.

9. Perrin, O., Godart, C., A model to support collaborative work in virtual enterprise. In Data \& Knowledge Engineering; $50: 63-86,2004$.

10.Teece, D.J., G. Pisano and A. Shuen, Dynamic Capabilities and Strategic Management, In Strategic Management Journal; 18(7), 1997. 